\title{
DISPARIDADES EN LAS CAPACIDADES DEL ESTADO LOCAL EN LA PROVINCIA DE SANTA FE. ¿OBSTACULO PARA INTRODUCIR REFORMAS SOCIALES?
}

\author{
Tesista: \\ Rodrigo Abelardo Müller \\ Directora de tesis: \\ Víctor Ramiro Fernández \\ Co-Directora de tesis: \\ María Jimena García Puentes
}

\section{IDENT IFICACIÓN DEL PROBLEMA}

El trabajo de tesis analiza la situación de las capacidades estatales de los Estados locales de la Provincia de Santa Fe en sus áreas de legitimación. Basado en numerosos aportes teóricos que sustentan que el Estado puede ser un actor autónomo y que dicho actor cuando cuenta con las capacidades necesarias, puede llevar adelante con eficacia las políticas públicas que se propone desarrollar. A diferencia de los tradicionales estudios enfocados en indagar sobre las áreas locales de acumulación me he centrado en las instancias de legitimación de los Estados locales. Así describo por un lado las razones dentro del escenario económico y social de estudiar los niveles locales como ámbito geográfico estratégico para cualquier proceso de desarrollo, tomando como base las investigaciones de la denominada "Nueva Ortodoxia Regionalista". Entre los fundamentos de tomar como análisis el ámbito de legitimación local es que el mismo aparece si bien como un ámbito separable analíticamente, pero empíricamente inescindible de los roles asignados a las políticas económicas. Por lo que al trasladarse a lo local el proceso de desarrollo, la responsabilidad de la problemática social también se traslada hacia dicho ámbito. Así en el trabajo se denotan los profundos cambios que han ocurrido en el ámbito de la legitimación para finalizar en los programas sociales socio-productivos emparentados con los principios de la nueva ortodoxiaregionalista y su ámbito de implementación en la esfera local. La vertiente teórica de la autonomía estatal descripta junto a la conexión entre lo local y el ámbito de la legitimación son las que dan pie desde lo teórico para avanzar en el estudio de los tres casos seleccionados.

\section{OBJETIVOS}

El objetivo general del trabajo es el de analizar el papel de las capacidades estatales en el campo del área de la legitimación en los Estados locales en la provincia de Santa Fe, comparando 
municipios del centro y del norte de la provincia, intentando comprobar en qué medida se puede verificar que "Los Estados locales de las ciudades con mayor dinamismo económico y cohesión social de la Provincia de Santa Fe muestran un relativo avance y escasas capacidades para el desarrollo de nuevas formas de gestión en las áreas de legitimación".

\section{METODOLOGÍA}

Para el trabajo se realizo un estudio de tres casos. Para ello se seleccionaron a las ciudades de Esperanza, Rafaela y Las Toscas. Esta última localidad ubicada en el norte de la provincia de Santa Fe, en el Departamento General Obligado lindando con la provincia del Chaco, y las demás (Rafaela), en el Departamento Castellanos, siendo la cabecera departamental y finalmente la ciudad de Esperanza como cabecera del Departamento Las Colonias (Esperanza). Cada una de ellas ha debido asumir un papel histórico en su región y, en especial en los departamentos donde se encuentran ubicadas. En tal sentido las ciudades de Esperanza y de Rafaela son cabeceras de su departamento, y se han mostrado -al menos discursivamente- con fuertes improntas que el Estado Municipal es el impulsor del desarrollo económico-social de sus ciudades. Las Toscas por su parte se encuentra entre las diez ciudades más importantes de la región norte de la Provincia de Santa Fe.

Tomando una vertiente de análisis estatal basada en una metodología histórica-comparativa, algunos autores han podido recuperar una visión acerca del Estado que puede remontarse a Max Weber. Rescatando a la organización estatal como un actor necesario para producir profundos cambios en las relaciones económicas-sociales de los países estudiados. Si bien con los estudios específicamente centrados en la industrialización de países ubicados en la periferia y en la comprobación de la tarea que le cupo al Estado como activo organizador de los recursos de la sociedad, financiador y regulador. A partir del papel del Estado como "solución" se intenta indagar, en cual es el grado de avance de las capacidades estatales locales a para involucrarse en políticas de legitimación.

Tomando la conceptualización de las capacidades estatales de Fernandez-Vigil como la "presencia o ausencia de determinadas estructuras organizacionales que se muestran críticas a la habilidad de las autoridades estatales para emprender determinada tareas" (Fernández, Vigil, 2007:1), se analizan las dimensiones interna y externa de las capacidades. Dichas capacidades son el producto de trayectorias históricas desarrolladas por las estructuras estatales, por lo que se asientan en la propia especificidad histórica de cada uno de los Estados nacionales (también regionales o locales) por lo que no es un rasgo estructural fijo de ningún sistema de gobierno (Skocpol, 2008), ya que la misma no es homogénea al interior de cada una las áreas estatales ni en la distribución sobre el territorio y cambian en el devenir de la historia de los Estados.

Por otro lado el insumo analítico para operacionalizar las categorías descriptas como capacidades estatales son los conceptos de capacidades internas: entendidas estas como un prerrequisito que denota la fortaleza del Estado en el ámbito de los recursos humanos, fiscales y un elevado nivel de organización de los mismos (Fernández, Vigil, 2007:4). Como segunda dimensión finalmente se aplica la variable de capacidades externas de los Estados municipales: constituidas por "la capacidad de penetrar la sociedad y desarrollar un alto poder operativo a través de procesos de "coproducción" con los sectores de la sociedad civil" (Fernandez, Güemes, Magnin, y Vigil, 2006:35).

El análisis de las capacidades internas de los Estados locales se desarrollo recurriendo a tres dimensiones de análisis compuestas por: el 
software, el hardware y el orgware (Fernández, Güemes, Magnin, y Vigil, 2006). El hardware compuesto de los recursos financieros individuales y comparados del Estado local, su carácter exógeno o endógeno; el software; de los recursos humanos del Estado local en las áreas vinculadas a la legitimación, analizando las formas de reclutamiento de los mismos, su formación y desarrollo de programas de capacitación en competencias generales y especificas y el orgware de la organización para producir insumos de planificación y gestión como las vinculaciones que se establecen entre el personal político y burocrático.

Por otra parte en las capacidades externas o infraestructurales: se analizan los mecanismos de implicación del Estado y los actores en el área de legitimación a través de la elección como objeto de análisis del "Programa Nacional Manos a la Obra", esto permite obsenvar las capacidades del Estado a nivel infraestructural en las tres localidades, para desarrollar este programa social cuyos postulados se encuentran próximos a las propuestas de desarrollo local de la nueva ortodoxia regionalista que también han penetrado las propuestas llevadas a cabo por un conjunto de políticas sociales.

\section{PRINCIPALES RESULTADOS}

Después de examinar de forma comparativa los Estados locales objeto de la investigación se puede apreciar que localidades con una situación socio-demográfica muy heterogénea entre si también cuentan con grado muy desigual de desarrollo de las capacidades estatales. Todas las Municipalidades distan de un tipo ideal, que permita decir que se cuentan con organizaciones con fuertes capacidades y el ejemplo más destacado de ello es el hecho de que ninguno de los Estados locales ha podido terminar con la reforma burocrática, ya por ejemplo los ingresos del personal se realizan por designaciones di- rectas y despreciando el mecanismo de ingreso por mérito al Estado municipal.

A pesar de ello desechando las recomendaciones incluso más intransigentes en materia de la Nueva Gestión Pública, se quiere avanzar introduciendo en algunos casos nuevos mecanismos de rendiciones de cuentas y auditorias ciudadanas como es el caso de Rafaela, sin haber agotado todas las posibilidades de haber terminado con la reforma jerárquica.

Este trabajo también demuestra para el caso de estas tres localidades las limitaciones que a nivel empírico presenta el Estado local para convertir a sus ciudades en comunidades cerradas, que por medio del impulso de políticas de legitimación para sus ciudadanos, las mismas se comporten como "islas" en la que sus índices socio-demográficos no puedan ser influidos; desde los condicionantes que aparecen desde el espacio macro-nacionales o macro-globales.

\section{CONCLUSIONES}

A través de la tesis se extraen una serie de conclusiones que subrayan en coincidencia con una línea crítica de la Nueva Ortodoxia Regionalista los límites que presentan las bondades de las teorías del desarrollo local. En el caso particular de la Provincia de Santa Fe el punto de partida del análisis demuestra profundas asimetrías socio-demográficas a nivel interdepartamental como es en la región norte e incluso al interior de la misma región centro. Por ello a partir de una escasa capacidad del Estados local de actuar como agente que pueda revertir una tradición de escaso industrialismo e índices que muestran una escasa integración social, que por otra parte responden a los vaivenes de políticas económicas-sociales nacionales y globales, es necesario reconocer los limites de los Estados locales para garantizar sociedades fuertemente inclusivas. 
Las ciudades de las Esperanza, Las Toscas y Rafaela presentan un recorrido inacabado en materia de capacidades estatales. Sera muy difícil alcanzar el éxito en cualquier programa que tenga como ejecutor a las instancias locales de gobierno si los tres casos que he analizado en este trabajo representan la generalidad de las Municipalidades de la Provincia de Santa Fe. Sin un software, un hardware y un orgware, como asimismo sin la debida inserción en la sociedad civil, ha quedado demostrado que los resultados que son posibles alcanzar -en este caso en el programa que hemos puesto como ejemplo como es el Programa Manos a la Obra- son insatisfactorios.
Cuando las instancias locales son tan reclamadas desde los estudios sociales como aquellas que llevaran a nuestras ciudades hacia la tierra prometida del desarrollo con inclusión social, nos encontramos con un panorama desolador en materia del "estado" del Estado Municipal en la Provincia de Santa Fe.

Por lo que los Estados locales resultan sometidos a que sus habitantes finalicen expuestos a la lógica de la "furiosa" competencia entre localidades por ganar un espacio dentro del concierto de los espacios exitosos. Romper las heterogeneidades hacia dentro de las regiones será posible siemprey cuando se dote de capacidades también a los niveles estatales locales.

\section{BIBLIOGRAFÍA}

Fernandez, V. R., Güemes, M. C.; Magnin, J. P. y Vigil, J. I. (2006): Capacidades Estatales y desarrollo regional. Realidades y desafíos para América Latina, Ediciones UNL, Santa Fe, Argentina.

Fernandez, V. R., Vigil J.I., (2007): “¿Cómo traer el Estado nuevamente al primer plano del desarrollo (regional) latinoamericano? Un estudio de las capacidades estatales al nivel meso-regional en la Argentina", Ponencia presentada al 4to. Congreso Argentino de Administración Pública, Sociedad, Gobierno y Administración, Ciudad Autónoma de Buenos Aires, 22, 23, 24, 25 de Agosto de 2007, Argentina.

Skocpol, T. (2008): “El Estado regresa al primer plano: Estrategias de análisis en la investigación actual", en Lecturas sobre el Estado y las Políticas Públicas. Retomando el debate de ayer para fortalecer el actual, Carlos Hugo Acuña, Jefatura de Gabinete de Ministros-Presidencia de la Nación, Buenos Aires, Argentina, pp. 169-202.

\section{PARA CITAR ESTE ARTÍCUL0:}

Müller, Rodrigo Abelardo (2011) "Recensión de tesis: 'Disparidades en las capacidades del estado local en la provincia de Santa Fe. ¿Obstáculo para introducir reformas sociales?'”, DAAPGE, año 11, N 17 , 2011, pp. 143-146. UNL, Santa Fe, Argentina. 